\section{Effect of the ingredient A97614A1 on the adhesion and biofilm formation of \\ Staphylococcus pseudintermedius in a model of reconstructed canine epidermis}

\section{Elodie Ollivier ${ }^{1}$, Claudine Zemirline ${ }^{1}$, Laetitia Marchand ${ }^{2}$, Brigitte Closs ${ }^{2}$}

1 Ceva Santé Animale, Libourne, France

2 Silab, Brive, France

\section{OBJECTIVES}

Dogs with atopic dermatitis present a loss of diversity of their skin microbiota with relative increases in Staphylococcus spp. abundances during flares. There is a strong correlation between this dysbiosis, inflammation and skin barrier defect. Staphylococcus pseudintermedius is also the most frequent pathogen isolated from skin and ear infections in dogs. The objective of this study was to evaluate the efficacy of the ingredient coded A97614A1 on the adhesion and biofilm formation of Staphylococcus pseudintermedius in a model of Reconstructed Canine Epidermis (RCE).

\section{METHODS}

Primary keratinocytes extracted from skin samples of two dogs were cultured in a specific medium for 12 days in order to develop RCEs. On day 12, RCEs were either treated topically with A97614A1 during 24 hours or not treated (controls). On day $13,50 \mu \mathrm{L}$ of a $106 \mathrm{CFU} / \mathrm{mL}$ suspension of Staphylococcus pseudintermedius isolated from a dog with pyoderma were topically applied on RCEs. Twenty-four hours later (day 14), RCEs were observed using scanning electron microscopy to assess the adhesion and biofilm formation of Staphylococcus pseudintermedius.

\section{RESULTS}

RCEs treated with A97614A1 presented much less Staphylococcus pseudintermedius than control RCEs. The ingredient A97614A1 therefore limited the adhesion and biofilm formation of Staphylococcus pseudintermedius at the surface of the RCEs.

\section{STATEMENT (CONCLUSIONS)}

These results support the potential interest of incorporating this ingredient into topical products intended for dogs presenting an alteration of their skin microbiota, including atopic dogs.

\section{Efficacy of the} ingredient A97614A1 in a model of reconstructed human epidermis stressed by cytokines

\section{Elodie Ollivier ${ }^{1}$, Claudine Zemirline ${ }^{1}$, Nicolas Amalric ${ }^{2}$, Valérie Rahoul ${ }^{2}$, Nadège Reymond $^{3}$, Marie-Christine Cadiergues ${ }^{4}$}

\footnotetext{
Ceva Santé Animale, Libourne, France

Synelvia, Labège, France

Consultant, Villeneuve Loubet, France

UDEAR, Université de Toulouse, INSERM, ENVT, Toulouse, France
}

\section{OBJECTIVES}

In dogs and cats as in humans, dermatological disorders are associated with impaired skin barrier, comprising a defective mechanical barrier, a disregulation of the immune response and an alteration of the skin microbiota. The objective of this study was to evaluate the efficacy of a new ingredient coded A97614A1 on the mechanical and immunological skin barrier, using an in-vitro model of Reconstructed Human Epidermis (RHE) stressed by cytokines to induce a skin barrier defect.

\section{METHODS}

RHE stressed by cytokines were treated with A97614A1 and compared to non-treated stressed RHE and to normal (not stressed and non-treated) RHE. The evaluation of the effect of A97614A1 on the mechanical skin barrier (including hydration) was assessed through the analysis of RHE morphology, outside-inside permeability (Lucifer yellow test), transepidermal water loss (TEWL), Natural Moisturising Factors (NMFs) and ceramides. The analysis of the pro-inflammatory cytokines IL- 8 and TSLP (markers of the immune response) served as criteria to evaluate the immunological skin barrier.

\section{RESULTS}

Compared to normal RHE, stressed RHE present a disorganisation of the structure (dissociation of cells, spongiosis, fewer and smaller keratohyaline granules), increased permeability and TEWL, and decreased amount of NMFs 
and ceramides; the IL- 8 and TSLP release is increased. Treatment with A97614A1 allowed stressed RHE to recover normal morphology, impermeability, TEWL, IL-8 and TSLP amounts, and close to normal amounts of NMFs and ceramides.

\section{STATEMENT (CONCLUSIONS)}

The ingredient A97614A1 was shown to have beneficial effects on the mechanical and immunological skin barrier. These results support the potential interest of incorporating it into topical products.

\section{Two case reports of novel cutaneous mycoses in cats in the UK using molecular identification for rapid and accurate diagnosis}

\section{Nikoleta Makri' ${ }^{1}$, Gavin Paterson ${ }^{2}$, Fiona Gregge ${ }^{3}$, Catriona Urquhart ${ }^{4}$, Holly McCluskey ${ }^{5}$, Tim Nuttall ${ }^{1}$}

1 Hospital for Small Animals, Royal (Dick) School of Veterinary Studies, Edinburgh, United Kingdom

2 Easter Bush Pathology, Royal (Dick) School of Veterinary Studies, Edinburgh, United Kingdom

3 Aberdeen PDSA Pet Hospital, Aberdeen, United Kingdom

4 Abervet, Aberdeen, United Kingdom

5 Riverside Veterinary Practice, Livingston, United Kingdom

\section{OBJECTIVES}

Phaeohyphomycosis and sporotrichosis are saprophytic mycoses affecting humans and animals. Traumatic inoculation results in cutaneous and subcutaneous lesions that can be locally invasive. Traditional culture based methods can be slow and may not sufficiently classify the fungi. Molecular testing however, can achieve a fast definitive identification.

\section{METHODS}

Two cats (cat 1-15-year-old male neutered domestic short hair; cat 2-11-year-old female neutered domestic long hair) developed focal suppurative nodular lesions on the dorsal nose. Both cats had been previously diagnosed with diabetes mellitus and were on insulin therapy. There was no response to broad spectrum antibiotics selected following antimicrobial susceptibility tests. Fungal elements were seen on cytology, although initial culture was negative in cat 2 . Subsequent isolates were obtained after overnight incubation of fresh discharge on Sabouraud dextrose agar with chloramphenicol. These were submitted for PCR and sequencing of the ribosomal internal transcribed spacers (ITS)with the primers ITS-F and ITS-R.

\section{RESULTS}

PCR and sequencing confirmed phaeohyphomycosis with Exophiala dermatitidis (cat 1) and sporotrichosis with Sporothrix pallida complex (cat 2).Further sequencing of calmodulin and beta-tubulin partial gene sequences confirmed Sporothrix humicola.

\section{STATEMENT (CONCLUSIONS)}

These are the first reports of Exophiala dermatitidis and Sporothrix humicola in cats in the UK. The use of molecular methods provided a rapid and definite identification of the fungi. Clinicians and pathologists need to be aware of the emerging use of molecular techniques to achieve rapid and accurate identification of rare fungal microorganisms. This will help reduce unnecessary antibiotic use.
Antimicrobial susceptibility of bacterial isolates from canine wounds in a referral population

\section{Catrina Pennington ${ }^{1}$, Tim Nuttall ${ }^{2}$, Jon Hall ${ }^{3}$}

1 University of Edinburgh, Edinburgh, United Kingdom 2 University of Edinburgh, Edinburgh, United Kingdom 3 University of Edinburgh, Edinburgh, United Kingdom

\section{OBJECTIVES}

To report the prevalence and antimicrobial susceptibility of bacteria isolated from canine wounds in a referral population.

\section{METHODS}

Data was collected from clinical records of dogs with wounds cultured between 01/01/2008 and 01/01/2018. Cases with incomplete clinical information or results were excluded. 1 isolate was included where the same bacteria were isolated from multiple samples in a dog.

\section{RESULTS}

392 cases (499 samples) were included. $83.5 \%$ of cultures were positive; $54.7 \%$ isolated a single organism and $28.8 \%$ two or more. 